\title{
Follicular metabolic changes and effects on oocyte quality in polycystic ovary syndrome patients
}

\author{
Yan Zhang ${ }^{2, *}$, Lingyan Liu ${ }^{1, *}$, Tai-Lang Yin ${ }^{4}$, Jing Yang ${ }^{4}$ and Cheng-Liang Xiong ${ }^{2,3}$ \\ ${ }^{1}$ School of Pharmaceutical Sciences, Capital Medical University, Beijing, China \\ ${ }^{2}$ Family Planning Research Institute/Center of Reproductive Medicine, Tongji Medical College, Huazhong University of Science \\ and Technology, Wuhan, Hubei Province, China \\ ${ }^{3}$ Wuhan Tongji Reproductive Medicine Hospital, Wuhan, Hubei Province, China \\ ${ }^{4}$ Reproductive Medicine Center, Renmin Hospital of Wuhan University, Wuhan, Hubei Province, China \\ *These authors contributed equally to this work and share co-first authorship \\ Correspondence to: Cheng-Liang Xiong, email: clxiong951@sina.com \\ Lingyan Liu, email: Lingyan@ccmu.edu.cn \\ Jing Yang, email: dryangqing@hotmail.com
}

Keywords: PCOS, follicular fluid, metabolomics, NMR, oocyte quality

Received: May 04, $2017 \quad$ Accepted: June 19, $2017 \quad$ Published: July 06, 2017

Copyright: Zhang et al. This is an open-access article distributed under the terms of the Creative Commons Attribution License 3.0 (CC BY 3.0), which permits unrestricted use, distribution, and reproduction in any medium, provided the original author and source are credited.

\section{ABSTRACT}

Polycystic ovary syndrome (PCOS) is a common complex and heterogeneous disorder, affecting up to $10 \%$ women at reproductive age. It causes three fourth of the ovulatory infertility and PCOS patients often give poor IVF quality. Although some metabolic profiles have been investigated in PCOS patient sera and urine, the follicular fluid, providing fruitful biochemical information about oocyte environment during development has been ignored. In this work, based on NMR metabolomics approach, metabolic profile of follicular fluid of PCOS patients has been explored and compared with healthy controls. Significant increases of glycoprotein, acetate, cholesterol, significant decreases of lactic acid, glutamine, pyruvate, and alanine, have been discovered in PCOS follicular fluids. Furthermore, the Pearson correlations analysis indicated significant relationship existed between ART results and NMR detected follicular metabolites. All these results indicated that PCOS may induce dyslipidemia, low-grade inflammation, and disorder of glycolysis, pyruvate and amino acid metabolism in follicular fluids.

\section{INTRODUCTION}

Polycystic ovary syndrome (PCOS) is a common endocrine and metabolic disorder affecting $5 \sim 10 \%$ of women at their reproductive age $[1,2]$. The clinical manifestations of this highly heterogeneous disease include amenorrhea, hirsutism, obesity, hyperinsulinemia, hyperandrogenism, polycystic ovaries via ultrasound, and it attributes three fourth of the ovulatory infertility [3]. Some researches even suggest a high risk of endometrial and ovarian cancer in PCOS [4]. In the meanwhile, oocytes collected from PCOS patients who undergo IVF are often of poor quality, leading to a high cancelation rate and low fertilization rate $[5,6]$. The main underlying pathophysiological mechanism was thought to be the abnormally increased androgen and/or insulin level. The obesity, in another way, performs as a synergistic effect in PCOS patients [7]. However, the complex etiology of PCOS, as well as how PCOS affects oocytes development, relating to both environmental and genetic factors, has not been fully understood yet.

Large advances referring "omics" technologies have been explored to further understand this complex disease, which aim to set up potential molecular diagnosis means for PCOS, to discover therapy indicators for effective management of the disease, to establish some molecular "ruler" for assessing oocyte quality, etc. Amongst them, metabolomics, comprehensively investigating fingerprints of all metabolites from a specific system is considered to be promising $[7,8]$. As the downstream products, 
metabolites summarize the final results of any biological events from both genes and proteins. Nevertheless, only a few researchers have focused on the area to discover biomarkers and explore the disease mechanism. Studies using NMR or/and GC-MS found carbohydrate, fat, and protein metabolism disturbance in PCOS plasma [9-12]. Additional abnormalities including saturated and unsaturated fatty acids, fatty acid amides, sulfated steroid, lysophosphatidylcholines, lysophosphatidyl ethanolamines, and carnitine were discovered by ultra-performance liquid chromatography coupled with mass spectrometry (UPLCMS) [13-16]. Almost all these articles studied on human sera, except one [16] looked into urine metabolites.

Follicular fluid is a non-negligible bio-fluid that plays a crucial role in reproductive functions. The follicular fluid provides the special environment for oocyte growth. It contains metabolites that are accumulated during the process. Meanwhile, these metabolites are critical for the oocyte development. Hence the follicular fluid can provide information about biochemical status of follicle [17]. The knowledge of metabolic information from PCOS patients' follicular fluid can provide some points of view on pathogenesis mechanisms in PCOS oocytes and how follicular metabolites affect oocytes quality in further [18]. However, follicular fluid has never been systematically studied in PCOS patients.

In this work, metabolic variance of follicular fluid from PCOS and healthy controls were discovered using ${ }^{1} \mathrm{H}$ NMR metabolomics approach. The correlation between follicular metabolites and oocyte quality based on the assisted reproductive techniques (ART) results were further evaluated. In comparison with PCOS serum metabolomics studies which aimed to discover diagnostic biomarkers, our goal is to explore pathogenesis mechanisms in PCOS oocytes environment and investigate the metabolic effects on oocyte quality.

\section{RESULTS}

\section{Clinical information of sample cohorts}

According to the clinical diagnosis, 15 patients with PCOS and 36 healthy controls were involved in this study. The clinical information in the form of mean \pm SD was shown in Supplementary Table 1. There was not any statistical difference between two sample groups in any ART results.

\section{Metabolic differences of follicular fluid between PCOS and controls}

Especially focusing on the small molecules, CPMG (Carr-Purcell-Meiboom-Gill) ${ }^{1} \mathrm{H}$ NMR spectra of follicular fluid provided a large number of identifiable peaks from metabolites. The ${ }^{1} \mathrm{H}$ NMR CPMG spectra averaged over follicular fluid samples from each of the PCOS and control cohorts, together with the average difference spectrum, were shown in Supplementary Figure 1. PCOS patients and healthy controls exhibited marked differences in some metabolite signals, including alanine, lactic acid, cholesterol, and pyruvate. The dimensional reduction analysis - PLS was applied to the NMR spectra to facilitate visualization of group difference. The two population cohorts presented separated trend in PLS score plot shown in Figure 1A. The OSC-PLS further filtered out confounding physiological variations unrelated to the disease, such as diet, age, etc. Clearer separated clusters were observed in OSC-PLS score plot (Figure 1B) as it only exhibited class related biological changes. Suggested by OSC-PLS loadings that decrease of glucose, pyruvate, alanine, lactate, and increase of acetoacetate, cholesterol etc. contributed to the cluster separation along latent variable 1 (LV1, Figure 1C).

To eliminate chemical noises, a total of 59 peak regions were identified, including amino acids, organic acids, glucose, choline and lipids. The chemical shifts and multiplicities of these 59 peaks were listed in Supplementary Table 2. Integrals of respective peak regions were also obtained. Subsequent analysis was employed on identified 59 metabolite peak regions. The PLS score plot based on these 59 peak regions presented a clearer separation (Supplementary Figure 2A). As suggested by loadings, acetate (PK45), glutamine (PK37, 42), and pyruvate (PK39) contributed most to the clusters (Supplementary Figure 2B).

To focus on the actual differences between two sample groups, $P$-values were calculated by Student's $t$-test (Supplementary Table 2). A total of 9 metabolite regions with $p<0.05$ showed the significance, including increased intensity of glycerol and lipid region, glycoprotein, acetate, cholesterol and decreased levels of lactic acid, glutamine, pyruvate, and alanine (Supplementary Figure 3). The corresponding contents of these 9 peak regions, together with another 8 regions with $p<0.1$ were shown in the heat map (Supplementary Figure 4). The different patterns can be visually observed in PCOS from healthy controls. All of these results indicated the existence of group wise difference in follicular fluids from the two sample cohorts.

\section{Metabolic pathway disturbance in PCOS follicles}

To explore the entire metabolic pathway map that fluctuated in PCOS follicles, all the metabolites with $p<0.05$ between two sample cohorts were imported into MetPA. All the matching pathways were plotted in Figure 2A according to their pathway impact values of pathway topology analysis and - $\log (\mathrm{p})$ of pathway enrichment analysis. In addition, the metabolites with $p$-value less than 0.1 were also included here to provide more information for a more complete pathway map in Figure 2B. The affected pathways included pyruvate metabolism, glycolysis, alanine, aspartate and glutamate metabolism etc. 


\section{Effects of metabolic perturbation on ART results}

To determine whether PCOS induced metabolic disturbance has effects on ART processes, Pearson correlation was calculated between each ART variables and 59 NMR metabolite regions respectively in different sample cohorts. The correlation coefficients (Figure 3A) and their significance in $p$-value (Figure 3B) illustrated that, in PCOS patients, different metabolic patterns were correlated with ART aspects. All significant correlations in different ART aspects were summarized in Table 1.

Significant correlation was observed in PCOS patients between their 2PN fertilization rate and several metabolites levels, including acetoacetate, acetate, 3 -hydroxuybutyrate, glycoprotein, $\beta$-glucose, and formic acid. Among them, only glucose was negatively correlated with fertilization results. While, no significant correlation with 2PN fertilization rate was found in control group. Interestingly, acetoacetate, acetate, 3-hydroxuybutyrate, and glycoprotein also exhibited certain levels of significant difference between PCOS and controls.

Significantly positive and negative correlations with cleavage rate were observed in formic acid and glucose respectively in PCOS patients. Both trends were similar to those of 2PN fertilization results above. Acetic acid level was negatively correlated with the cleavage results in controls. This metabolite was also found important in fertilization step as above; however, it was positively correlated with 2PN fertilization rate in PCOS. Results showed that acetic acid plays negative role in the cleavage step in control group, while it plays positive role in the fertilization step in PCOS group.

Creatine, leucine, isoleucine, and tyrosine were highly negatively correlated with embryos quality in all samples (Supplementary Figure 5). While, the correlations were weakened in PCOS sample cohort. In comparison, histidine exhibited highly negative correlation and glutamine presented highly positive correlation with embryos top-quality rate respectively only in PCOS patients (Figure 4). The ANOVA suggested significant linear relationship between embryos top-quality rate and both metabolites respectively. All these observations indicated that PCOS induced metabolic perturbations affect ART process.

\section{DISCUSSION}

PCOS is a heterogeneous disorder with different phenotypes in affected women. In this study, we are the first to apply NMR to investigate changes of metabolic profile in PCOS follicular fluids. The changes in the levels of follicular metabolites reflected abnormal metabolic pathway fluctuation that related to physiological functions. Moreover, it is important to explore the intra-follicular metabolites pattern because PCOS patients undergoing IVF always contain morphologically normal metaphase II oocytes but have impaired oocyte development [19].

Different from the findings of metabolites in PCOS plasma [11, 13], pyruvate and lactate were significant decreased, together with increased level of acetate in
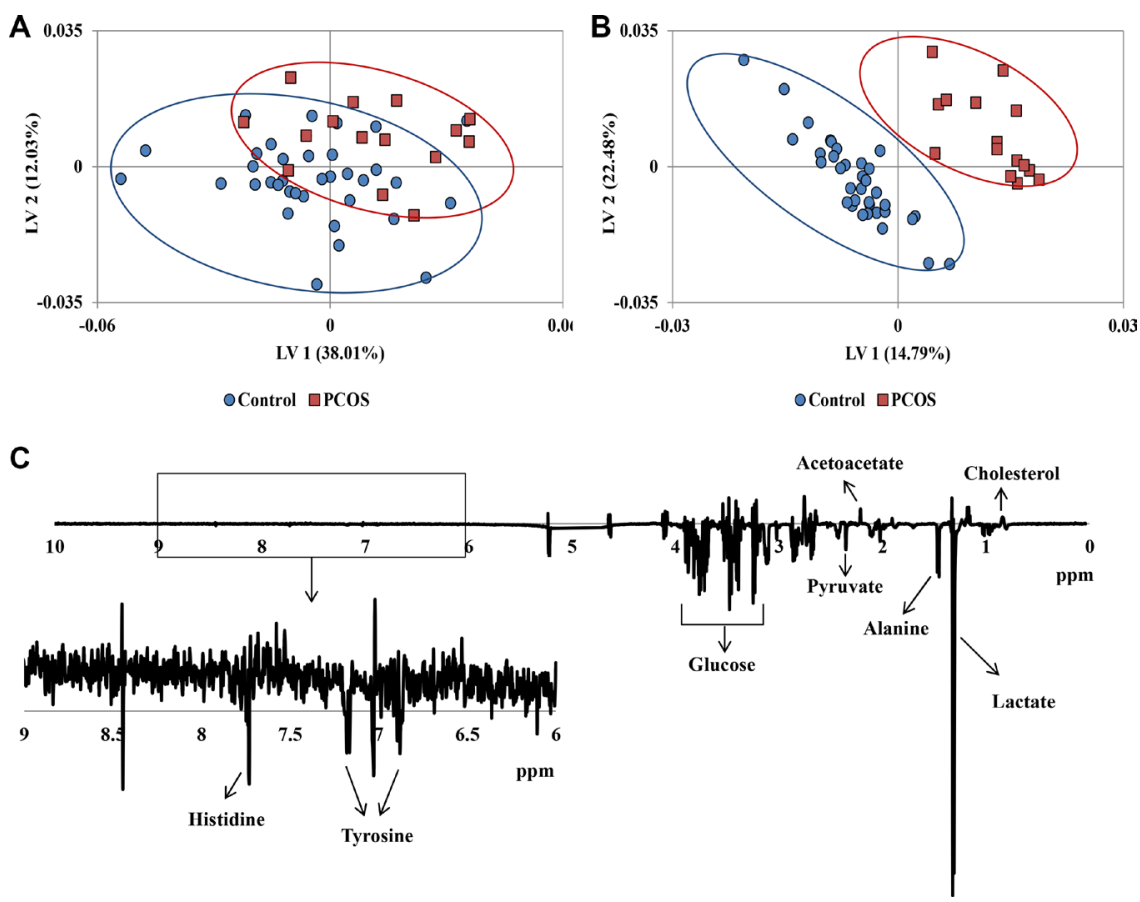

Figure 1: (A) PLS score plot of ${ }^{1} \mathrm{H}$ NMR CPMG spectra of all samples. (B) OSC-PLS score plot of ${ }^{1} \mathrm{H}$ NMR CPMG spectra of all samples. (C) Loadings plot on latent variable 1 (LV1) for OSC-PLS analysis of the whole CPMG spectral data. 

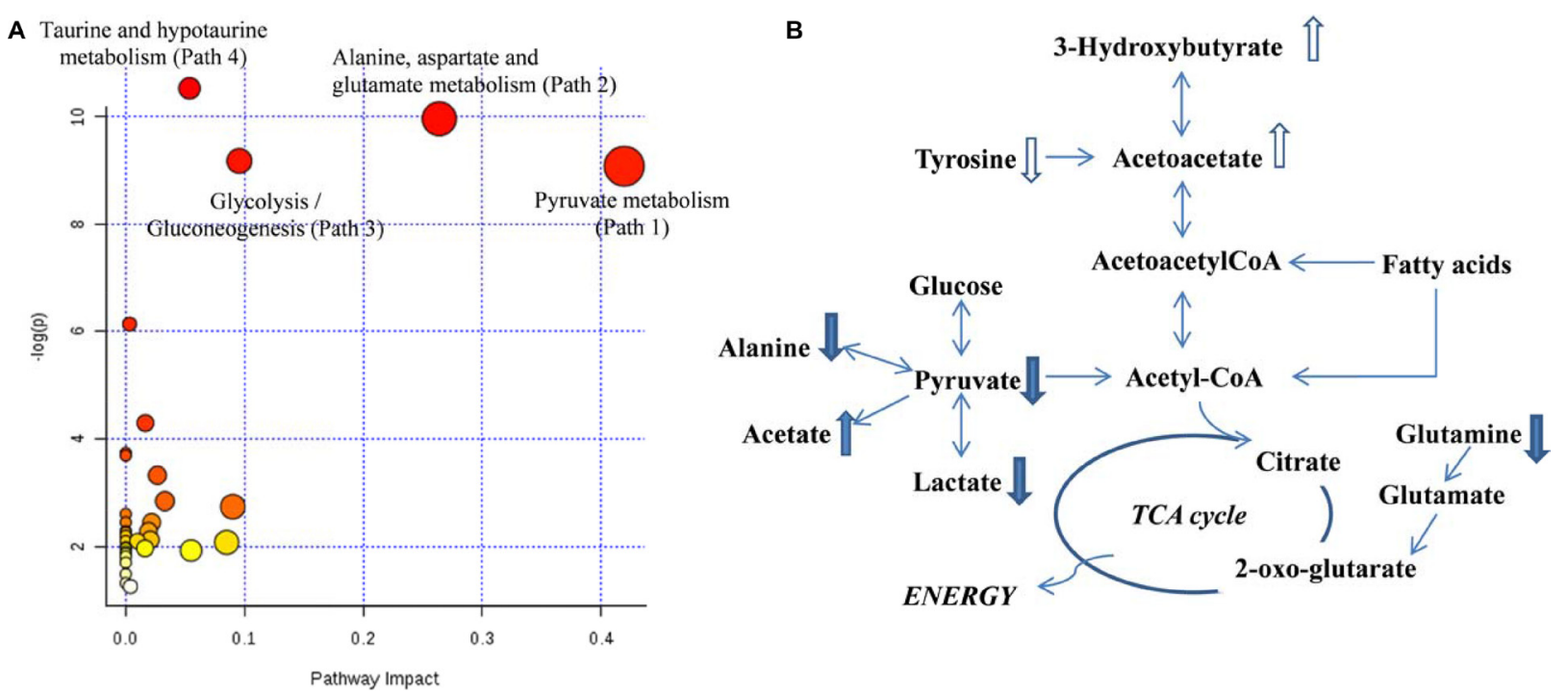

Figure 2: (A) Overview of altered metabolisms suggested by MetPA; (B) Pathway diagram showing altered metabolites in PCOS follicular fluids. Upward (downward) arrows indicate slightly higher (lower) levels $(p<0.1)$ in PCOS. The solid filled upward (downward) arrows indicate significantly higher (lower) levels $(p<0.05)$ in PCOS.

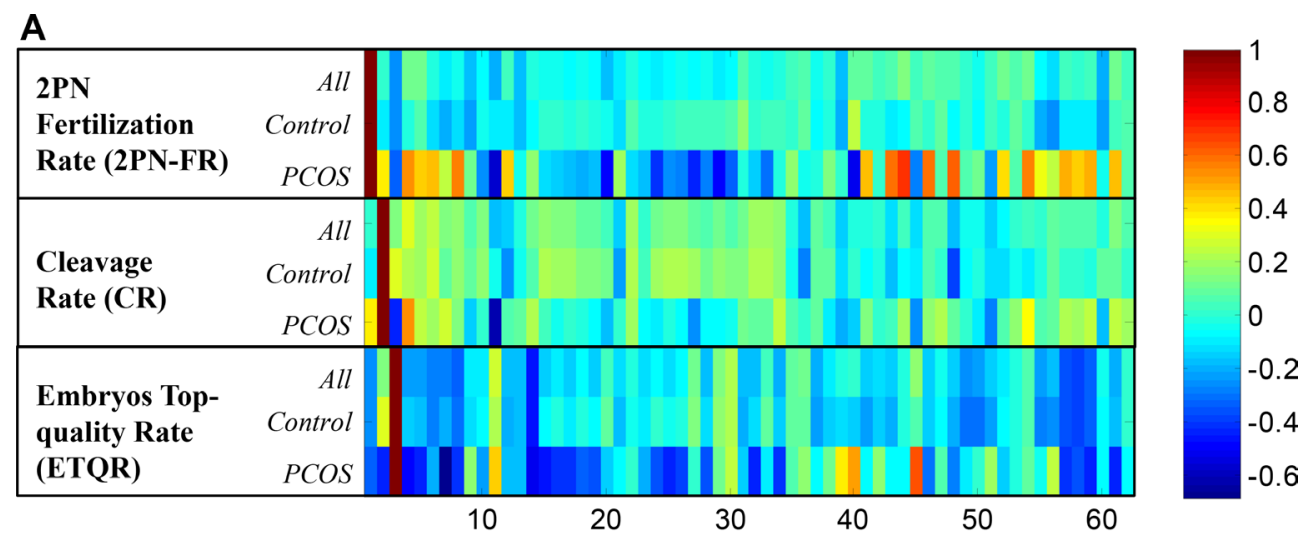

B

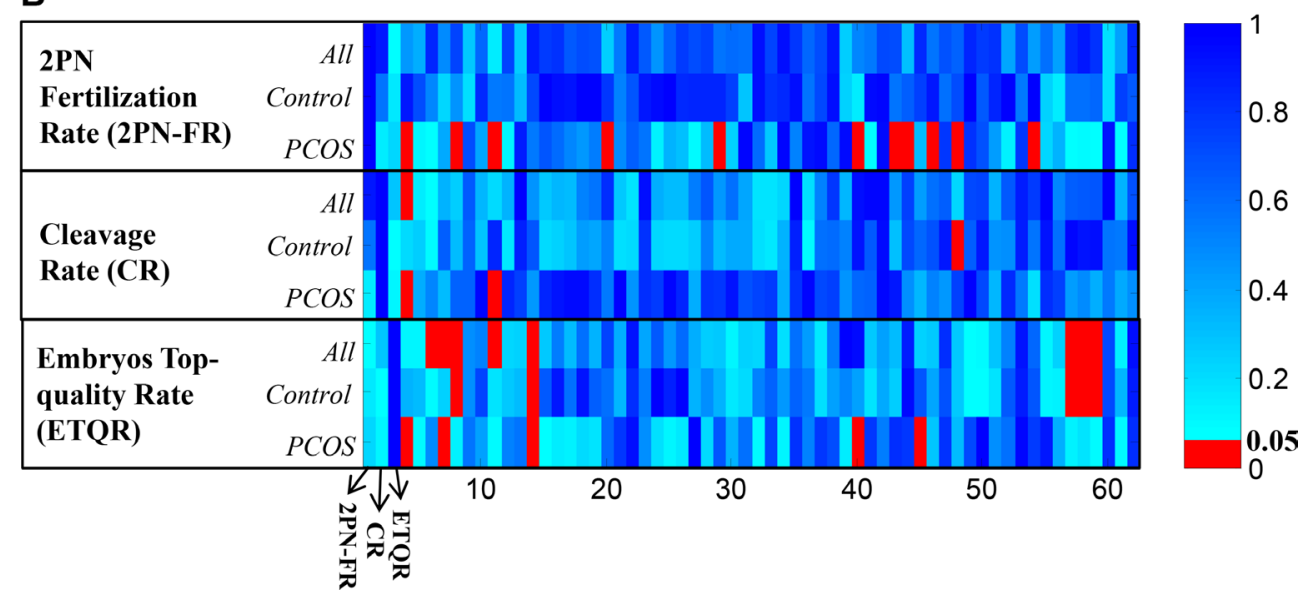

Figure 3: (A) Pearson correlation coefficients between ART clinical results (2PN fertilization rate, cleavage rate, and embryos top-quality rate) and NMR detected peak regions. The columns represent the $2 \mathrm{PN}$ fertilization rate, cleavage rate, top-quality embryos rate (x-axis $1 \sim 3$ ), and PK1 to PK59 (x-axis 4 62) respectively; (B) Pearson correlation $p$-values of each paired data. 
Table 1: Pearson correlation coefficients between NMR detected follicular metabolites and ART results in all samples, PCOS patients, and healthy controls respectively

\begin{tabular}{lccccc}
\hline Peak ID & Metabolites & All & Controls & PCOS & PCOS and control $p$-value \\
\hline \multicolumn{2}{l}{ 2PN Fertilization Rate } & & & & \\
PK41 & Acetoacetate & 0.15 & -0.08 & $0.69(4.5 \mathrm{E}-3)$ & 0.078 \\
PK45 & Acetate & 0.07 & -0.06 & $0.63(0.012)$ & $2.5 \mathrm{E}-3$ \\
PK40 & 3-hydroxuybutyrate & 0.05 & -0.10 & $0.60(0.017)$ & 0.060 \\
PK43 & Glycoprotein & 0.08 & 0.021 & $0.59(0.020)$ & 0.020 \\
PK8 & B-glucose & -0.21 & -0.10 & $-0.57(0.026)$ & \\
PK1 & Formate & 0.11 & -0.02 & $0.55(0.036)$ & \\
PK5 & Tyrosine & -0.05 & -0.13 & $0.55(0.032)$ & \\
Cleavage Rate & & & & & \\
PK8 & B-glucose & -0.18 & -0.03 & $-0.61(0.016)$ & \\
PK1 & Formate & $0.30(0.035)$ & 0.21 & $0.55(0.035)$ & \\
PK45 & Acetate & -0.17 & $-0.37(0.025)$ & 0.09 & \\
Embryos Top-quality Rate & Creatine & $-0.46(6.7 \mathrm{E}-4)$ & $-0.45(5.5 \mathrm{E}-3)$ & $-0.53(0.044)$ & \\
PK11 & Leucine & $-0.38(6.5 \mathrm{E}-3)$ & $-0.39(0.018)$ & -0.36 & \\
PK55 & Isoleucine & $-0.36(0.01)$ & $-0.36(0.029)$ & -0.40 & \\
PK54 & Tyrosine & $-0.32(0.022)$ & $-0.31(0.06)$ & -0.40 & \\
PK5 & Histidine & $-0.29(0.040)$ & -0.20 & $-0.68(4.8 \mathrm{E}-3)$ & \\
PK4 & Glutamine & 0.17 & 0.07 & $0.65(8.7 \mathrm{E}-3)$ & \\
PK42 & Pear & & & \\
\hline
\end{tabular}

In parentheses, the Pearson correlation $p$-value was illustrated if it is less than 0.05 .
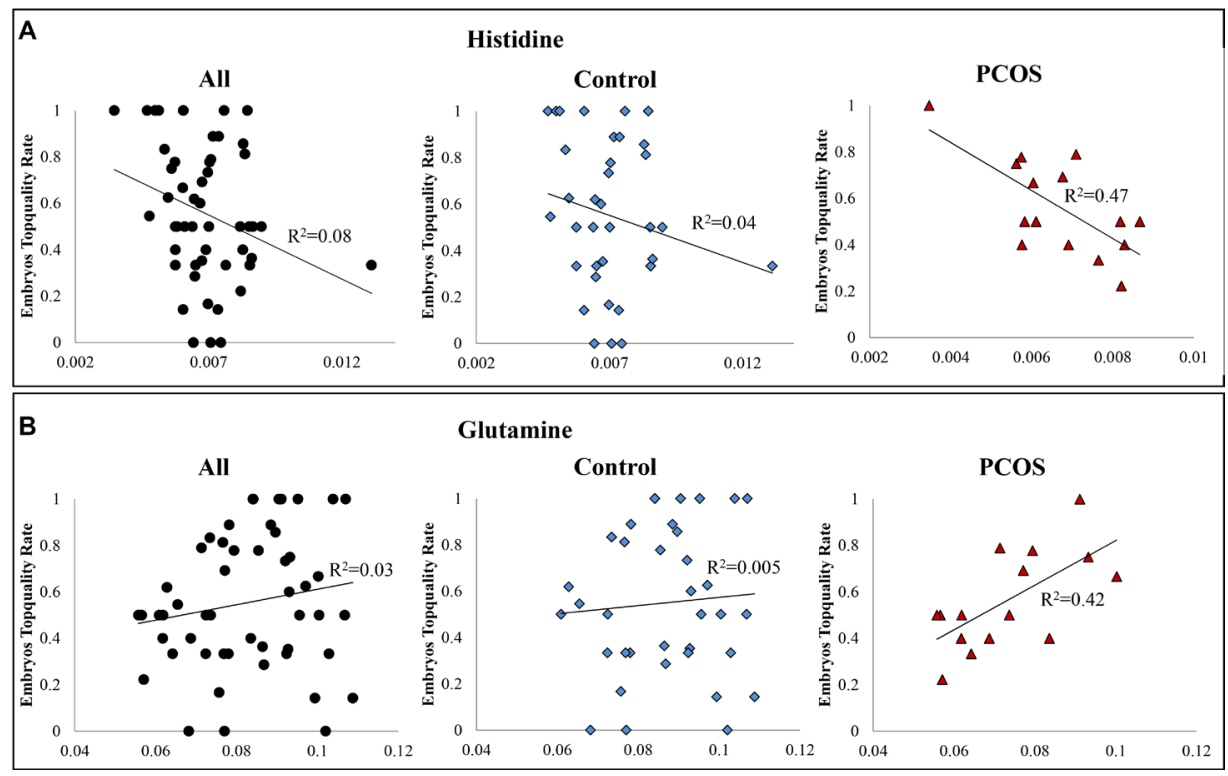

Figure 4: (A) Scatter plot of embryos top-quality rate and histidine. A significantly negative correlation exists between intrafollicular histidine level and embryos top-quality rate in PCOS patients $\left(\mathrm{y}=-103.36 \times+1.25, R^{2}=0.47, p<0.005\right)$; (B) Scatter plot of embryos top-quality rate and glutamine. A significantly positive correlation exists between intra-follicular glutamine level and embryos top-quality rate in PCOS patients $\left(\mathrm{y}=9.62 \times-0.14, R^{2}=0.42, p<0.009\right)$. X-axis was abundance of the metabolite in NMR spectra (integral of peak region); y-axis was the embryos top-quality rate. 
follicular fluids of PCOS patients. This indicated the alteration in pyruvate metabolism and glycolysis, which were highlighted by the impact value of 0.42 and 0.1 respectively in Figure 2A. It suggested impaired glucose mechanism were found in granulosa-lutein cells from women with polycystic ovaries [20]. The abnormal insulin level contributes to the decrease of glucose uptake and related metabolism in PCOS ovary and follicles. Central insulin resistance could be one but not the only reason of hyperinsulinemia in PCOS [21]. Those regulators affecting insulin functions are also unusually expressed in PCOS ovaries, for example, insulin resistance associated phosphatidylinositol 3-phosphate kinase (PI3-K) is downregulated. Similarly, the down-regulated annexin A2 suppresses the expression of glucose transporter GLUT-4, leading to the deficiency of glucose transportation [22]. It is also supported by other studies that some involving pathways like glycolysis which have been found downregulated in endometrium of PCOS women [23]. From our study, although the abnormal glucose level was not observed in PCOS follicular fluids, a significantly negative correlation of glucose with both fertilization rate and cleavage rate were found in PCOS. It is also suggested by other studies that, the fertilization [24] and cleavage [25] steps are quite glucose uptake dependent.

A slightly increase of acetoacetate and 3-hydroxybutyrate was observed in PCOS follicular fluids at meanwhile. These ketone body products come from the up-regulated fatty acid degradation. It is probably that alternative energy pathways were provoked to compensate the impairment of pyruvate metabolism and glycolysis for energy demanding [7]. The acetoacetate and 3-hydroxybutyrate were also positively correlated with 2PN fertilization rate in PCOS patients. While, this significant correlation was not found in control group. These ketone bodies were crucial as energy substrates in early embryo development [26]. It further supported that the energy alternatives were raised because of PCOS.

Similarly, significant decrease of alanine and glutamine, as well as a slight decrease of tyrosine in PCOS follicular fluids demonstrated an alteration in amino acid metabolism. It is likely that amino acids were increasingly consumed to provide energy. However, glutamine was found to be positively correlated with embryos top-quality rate only in PCOS. This was agreed with that glutamine and lactate were found positively correlated, and lactate was decreased in oocytes fertilization and cleavage steps [25]. It may indicate, with deficient energy source, the amount of energy alternative was crucial for embryo development.

It is not surprising that a significantly increased level of glycerol and lipid region (Pk18), cholesterol and a slightly higher level of lipids (LDL) were present in PCOS follicular fluids. It was the same with the findings in PCOS serum from both NMR and MS studies, in which levels of other free fatty acids like palmitoleic acid, linoleic acid etc. raised too [21]. These are typical signs of dyslipidemia, which characterized by a decrease in HDL, an increase in LDL and triglycerides in PCOS. This is due to the impaired lipase expression and altered lipolysis caused by insulin resistance [27].

Glycoprotein was significantly increased in PCOS follicular fluids. This was in the same trend as observed in PCOS sera [11]. Evidence has been shown that glycoprotein is closely related to various inflammatory disorders, including infection, cardiovascular disorder, diabetes, etc [28]. The finding in our study indicated oocytes' environment may be related to low-grade inflammation in PCOS patients. Such intra-follicular inflammation was also found in PCOS patients from other studies. The altered GC expression of cytokines, chemokines, and immune cell biomarkers is possibly the main cause [29].

Beside, an interesting finding was that, in all follicular fluid samples, significantly negative correlations appeared between embryos top-quality rate and several amino acids, including creatine, leucine, isoleucine, as well as tyrosine. The correlations with first three amino acids were even significant in control group. Although not known by underlying mechanism, it is in agreement with a study that 11 amino acids (including isoleucine, etc.) were found elevated in follicular fluids from patients with repeated IVF failure [30].

Despite metabolic difference that was observed in PCOS follicular fluid, as well as interesting findings that explained the correlation between follicular fluid metabolites and ART outcomes, some limitations needed to be addressed in the study. Firstly, the relatively small number of available samples in this pilot study may limit the statistical power to discovery small changes in the metabolic profile. Further validation of different sample cohorts will be expected to draw a clearer picture. In addition, this exploratory study limited the investigation on small molecules with relatively high contents detectable by NMR. A deeper observation using more sensitive techniques will be expired in future studies.

\section{MATERIALS AND METHODS}

\section{Follicular fluid samples}

Human follicular fluid samples were obtained from the Renmin Hospital of Wuhan University. The study was approved by the Institutional Review Boards. All 15 PCOS patients were diagnosed according to the criteria from both the European Society of Human Reproduction and Embryology and the American Society for Reproductive Medicine in 2003 [31]. The 36 healthy controls were from the women under reproductive age who participated in ART because of their couples' sterility. All individuals from both sample cohorts were Chinese females and from the same province (Hubei) in China, which means they had the similar diet and life style. All the samplings were carried out in the mornings, and appropriate diet was suggested 
before oocyte retrieval. The same ovarian stimulation protocol was applied. Oocytes were retrieved using transvaginal ultrasound under guidance 34-36 hours after HCG administration. Oocytes were collected. Follicular fluid from same patient was combined, transported under dry ice and stored at $-80^{\circ} \mathrm{C}$ until analysis.

\section{Clinical information}

In 3 hours after oocyte retrieval, insemination was conducted with Percoll-prepared spermatozoa, whose population of motile spermatozoa was more than 7000-10000/ml. Subsequently, oocytes were transferred to a fresh medium. The occurrence of 2 pronuclei (PN) was observed and the number was recorded. The $2 \mathrm{PN}$ fertilization rate was calculated by dividing $2 \mathrm{PN}$ counts by oocyte retrieved number. After syngamy, the ratio of cleavage number to the fertilization number was calculated as cleavage rate. The specific evaluation standard for embryo quality on 3 days after cleavage was as follows: grade I, embryos with equivalent size and shape, and no obvious fragments were found; grade II, with slightly different size and shape, the percentage of DNA fragment was less than $20 \%$; grade III, with significantly different size and the DNA fragment was between 20\% and 50\%; grade IV, DNA fragment was more than 50\%. Embryos with good quality were defined as the embryos on day 3 belong to grade I or II, and the number of blastomeres were 6 to 9. Finally, the top-quality embryo rate was calculated as the ratio of good quality embryo number to the $2 \mathrm{PN}$ cleavage number.

\section{NMR experiments}

Each frozen follicular fluid sample was thawed, vortexed, centrifuged and then $530 \mu \mathrm{L}$ was transferred to 5-mm NMR tubes. A co-axial capillary containing $60 \mu \mathrm{L}$ TSP $(0.53 \mathrm{mmol})$ in D2O was placed into the NMR tube to serve as chemical shift and quantitative reference. The samples were randomized before performing the NMR experiments. All ${ }^{1} \mathrm{H}$ NMR experiments were carried out at a $25^{\circ} \mathrm{C}$ on a Bruker DRX500-MHz NMR spectrometer. ${ }^{1} \mathrm{H}$ NMR data for each sample was acquired using CPMG (Carr-Purcell-Meiboom-Gill) pulse sequences. Water was suppressed using a presaturation pulse. For each spectrum, 64 transients were collected and $16 \mathrm{~K}$ data points were acquired using a spectral width of $6000 \mathrm{~Hz}$. An exponential weighting function corresponding to $0.5 \mathrm{~Hz}$ line broadening was applied to the free-induced decay before Fourier transformation. Phasing and baseline correction were applied using Bruker Xwinnmr software version 3.5.

\section{Data analysis}

The NMR spectra obtained using the CPMG sequence were devoid of broad peaks from macromolecules and hence were more suitable for investigating altered levels of metabolites. The datasets were aligned with reference to the alanine peak at 1.46 ppm using MestReNova version 6.6.1. Each CPMG NMR spectrum was binned to $4 \mathrm{k}$ frequency buckets of equal size $(0.003 \mathrm{ppm})$. The spectra were then normalized with reference to the total spectra integral $(\delta$ $10.0 \sim 0.40 \mathrm{ppm}$ ), excluding the residual water and urea region $\delta 4.7 \sim 5.2 \mathrm{ppm}$. The spectral data were initially mean-centered and subjected to supervised multivariate statistical analysis including partial least-squares analysis (PLS) and orthogonal-signal-corrected partial leastsquares (OSC-PLS) analysis.

In a more targeted analysis, a total of 59 representative peak regions were identified according to the Human Metabolome Data Base and the comprehensive NMR study of follicular fluid [32]. Integrals for these peak regions were obtained and standardized to a sample mean of 0 and standard deviation of 1 to avoid any magnitude influences. Subsequently, these 59 metabolite signals were subjected multivariate analysis, Student's $t$-test, and correlation analysis with ART clinical data using Matlab (R2008a; Mathworks, Natick, MA) and PLS Toolbox (version 4.11, Eigenvector Research Inc.). The heat map and ANOVA result of linear regression fitting was constructed by R (version 3.3.1).

\section{CONCLUSIONS}

The present study is a pioneer to investigate metabolic variations in PCOS follicular fluid based on NMR metabolomics approach. The differential metabolites indicated dyslipidemia and main alternations in pyruvate metabolism, glycolysis, amino acid metabolism, and inflammation. The findings provided deep insight for PCOS pathogenesis mechanisms that happened in oocytes environment. Furthermore, the metabolic effects on oocytes quality were firstly explored based on the ART results, which suggested embryo top-quality rate was closely correlated with creatine, leucine and isoleucine in all follicular fluids, while specifically related to histidine and glutamine only in PCOS patients. These results provided us a better knowledge of human oocyte physiology, and metabolic effects on oocyte quality. Future work would be expected to increase the sample number for further validation, and to explore more subtle metabolic variation using techniques with higher sensitivity.

\section{Authors' contributions}

Y. Zhang and L. Liu contributed equally to this work. Y. Zhang, L. Liu, and T. L. Yin, conducted the experiments; Y. Zhang, and L.Liu analyzed the data, L. Liu J. Yang and C. X. Xiong designed the experiments, L. Liu, wrote the manuscript. All authors reviewed the manuscript. 


\section{ACKNOWLEDGMENTS}

This work was supported by grants from Beijing Municipal Education Commission Foundation, China (No.KM201610025008), Health and Family Planning Commission of Hubei Province, China [No. WJ2015Q017], and National Natural Science Foundation of China [No.81401276].

\section{CONFLICTS OF INTEREST}

All authors declare no conflicts of interests.

\section{REFERENCES}

1. Azziz R, Woods KS, Reyna R, Key TJ, Knochenhauer ES, Yildiz BO. The Prevalence and Features of the Polycystic Ovary Syndrome in an Unselected Population. J Clin Endocr Metab. 2004; 89:2745-9.

2. Goodarzi MO, Dumesic DA, Chazenbalk G, Azziz R. Polycystic ovary syndrome: etiology, pathogenesis and diagnosis. Nat Rev Endocrinol. 2011; 7:219-231.

3. Kini S. Polycystic ovary syndrome: diagnosis and management of related infertility. Obstetrics Gynaecology \& Reproductive Medicine. 2012; 22:347-353.

4. Dumesic DA, Lobo RA. Cancer risk and PCOS. Steroids. 2013; 78:782-5.

5. Heijnen EM, Eijkemans MJ, Hughes EG, Laven JS, Macklon NS, Fauser BC. A meta-analysis of outcomes of conventional IVF in women with polycystic ovary syndrome. Hum Reprod Update. 2005; 12:13-21.

6. Vrtačnik-Bokal E, Virant-Klun I, Meden-Vrtovec H. Quality of oocytes and embryos in patients with polycystic ovaries. International Congress. 2004; 1271:112-115.

7. Arya BK, Haq AU, Chaudhury K. Oocyte quality reflected by follicular fluid analysis in poly cystic ovary syndrome (PCOS): a hypothesis based on intermediates of energy metabolism. Med Hypotheses. 2012; 78:475-8.

8. Murri M, Insenser M, Escobar-Morreale HF. Metabolomics in polycystic ovary syndrome. Clin Chim Acta. 2014; 429:181-188.

9. Atiomo W, Daykin CA. Metabolomic biomarkers in women with polycystic ovary syndrome: a pilot study. Mol Hum Reprod. 2012; 18:546-53.

10. Escobarmorreale HF, Samino S, Insenser M, Vinaixa M, Luqueramírez M, Lasunción MA, Correig X. Metabolic heterogeneity in polycystic ovary syndrome is determined by obesity: plasma metabolomic approach using GC-MS. Clin Chem. 2012; 58:999-1009.

11. Sun L, Hu W, Liu Q, Hao Q, Sun B, Zhang Q, Mao S, Qiao J, Yan X. Metabonomics reveals plasma metabolic changes and inflammatory marker in polycystic ovary syndrome patients. J Proteome Res. 2012; 11:2937-46.

12. Yue Z, Li F, Rong L, Wang LN, Yan Y, Liu NN, Zhang CM, Ying W, Ping L, Tu BB. Metabolic profiles characterizing different phenotypes of polycystic ovary syndrome: plasma metabolomics analysis. BMC Med. 2012; 10:1-12.

13. Zhao X, Xu F, Qi B, Hao S, Li Y, Li Y, Zou L, Lu C, Xu G, Hou L. Serum Metabolomics Study of Polycystic Ovary Syndrome Based on Liquid Chromatography-Mass Spectrometry. J Proteome Res. 2014; 13:1101-11.

14. Fang D, Dan D, Chen H, Wei C, Li Q, Rong L, Ding S. Serum metabolomics study of polycystic ovary syndrome based on UPLC-QTOF-MS coupled with a pattern recognition approach. Anal Bioanal Chem. 2015; 407:4683-4695.

15. Chen YX, Zhang XJ, Huang J, Zhou SJ, Liu F, Jiang LL, Chen M, Jian-Bo W, Yang DZ. UHPLC/Q-TOFMS-based plasma metabolomics of polycystic ovary syndrome patients with and without insulin resistance. J Pharm Biomed Anal. 2016; 121:141-150.

16. Wang W, Wang S, Shan T, Wen M, Qian Y, Zeng X, Guo Y, Chao Y. Detection of urine metabolites in polycystic ovary syndrome by UPLC triple-TOF-MS. Clin Chim Acta. 2015; 448:39-47.

17. Dumesic DA, Meldrum DR, Katzjaffe MG, Krisher RL, Schoolcraft WB. Oocyte environment: follicular fluid and cumulus cells are critical for oocyte health. Fertil Steril. 2015; 103:303-316.

18. Revelli A, Piane LD, Casano S, Molinari E, Massobrio M, Rinaudo P. Follicular fluid content and oocyte quality: from single biochemical markers to metabolomics. Reprod Biol Endocrinol. 2009; 7:1-13.

19. Dumesic DA, Padmanabhan V, Abbott DH. Polycystic ovary syndrome and oocyte developmental competence. Obstet Gynecol Surv. 2008; 63:39-48.

20. Rice S, Christoforidis N, Gadd C, Nikolaou D, Seyani L, Donaldson A, Margara R, Hardy K, Franks S. Impaired insulin-dependent glucose metabolism in granulosa-lutein cells from anovulatory women with polycystic ovaries. Hum Reprod. 2005; 20:373-81.

21. Dong F, Deng D, Chen H, Cheng W, Li Q, Luo R, Ding S. Serum metabolomics study of polycystic ovary syndrome based on UPLC-QTOF-MS coupled with a pattern recognition approach. Anal Bioanal Chem. 2015; 407:4683-4695.

22. Ma X, Fan L, Meng Y, Hou Z, Mao YD, Wang W, Ding W, Liu JY. Proteomic analysis of human ovaries from normal and polycystic ovarian syndrome. Mol Hum Reprod. 2007; 13:527.

23. Kim J, Song H, Kang HH, Jun J, Hong S, Koong M, Kim I. Transcriptional profiling with a pathway-oriented analysis identifies dysregulated molecular phenotypes in the endometrium of patients with polycystic ovary syndrome. J Clin Endocrinol Metab. 2009; 23:278.

24. Dangoor M, Sasson S, Davarashvili A, Almagor M. Expression of glucose transporter and glucose uptake in human oocytes and preimplantation embryos. Hum Reprod. 1997; 12:2508-10.

25. Wallace M, Cottell E, Gibney MJ, Mcauliffe FM, Wingfield M, Brennan L. An investigation into the relationship between the metabolic profile of follicular fluid, 
oocyte developmental potential, and implantation outcome. Fertil Steril. 2012; 97:1078.

26. Gómez E. Acetoacetate and $\beta$-d-hydroxybutyrate as energy substrates during early bovine embryo development in vitro. Theriogenology. 1997; 48:63-74.

27. Teede H, Deeks A, Moran L. Polycystic ovary syndrome: a complex condition with psychological, reproductive and metabolic manifestations that impacts on health across the lifespan. BMC Med. 2010; 8:41.

28. Schultz DR, Arnold PI. Properties of four acute phase proteins: C-reactive protein, serum amyloid A protein, alpha 1-acid glycoprotein, and fibrinogen. Seminars in Arthritis \& Rheumatism. 1990; 20:129-47.

29. Adams J, Liu Z, Ren YA, Wun WS, Zhou W, Kenigsberg S, Librach C, Valdes C, Gibbons W, Richards J. Enhanced Inflammatory Transcriptome in the Granulosa Cells of Women with Polycystic Ovarian Syndrome. J Clin Endocr Metab. 2016; 101:jc20154275.
30. Xia L, Zhao X, Sun Y, Hong Y, Gao Y, Hu S. Metabolomic profiling of human follicular fluid from patients with repeated failure of in vitro fertilization using gas chromatography/mass spectrometry. Int J Clin Exp Pathol. 2014; 7:7220-9.

31. Rotterdam ESHRE/ASRM-Sponsored PCOS Consensus Workshop Group. Revised 2003 consensus on diagnostic criteria and long-term health risks related to polycystic ovary syndrome. Hum Reprod. 2004; 81:19-25.

32. Piñero-Sagredo E, Nunes S, de Los Santos MJ, Celda B, Esteve V. NMR metabolic profile of human follicular fluid. NMR Biomed. 2010; 23:485-495. 